\title{
On Body Language During Cello Performance
}

\author{
Jing Yuan
}

\author{
School of Music Shaanxi Normal University
}

Key words: Cello; Performance; Body; Language

\begin{abstract}
In the performance of cello, performers' body language is also an expression form. Appropriate and unique body language can add quite a lot splendor for the performance of cello. Different cello players have different performance styles as well as different viewpoints and understanding of music, so body languages during cello play are also different. Cello players' body language can help them express their feelings and present their styles; it also contributes to the usage of performance techniques. This paper explains the importance of body language to cello play and analyzes the application methods and characteristics of body language in cello play; some viewpoints and opinions on how to apply body language appropriately in cello play are also proposed. Appropriate body language is harmonious and beautiful if it integrates with cello play. Therefore, aesthetic principles must be observed when body language is added into cello play. Cello players' body language is also a kind of language. In the performance process, appropriate body language and facial expressions must be applied to integrate with music and bring audiences into the situation of the music so that audiences can enjoy beauty. If cello players keep dull expressions and rigid movements in the performance, the performance effects would be influenced, and audiences' appreciation of music would also be influenced so that they cannot enjoy the music well. Therefore, cello players' body language is critical to the expression of music emotions, and it can strengthen the expressive force of cello music. Cello players' movements and facial expressions are also a part of the performance, and they are a very important part in cello play.
\end{abstract}

\section{Introduction}

Body language is very important in cello play, since it can help music present its unique charm. There was such a story in the Warring States Period. When a music master finished playing, the guests did not understand it completely, so they hoped that the master would explain it. However, the master did not obey the guests; rather, he played the music from beginning to the end again. This story indicates the timeliness and mobility of music. Music language is very unique. It cannot be explained and elaborated with language. People can only arouse their inner resonance by feeling music on the scene, appreciating performers' performance, feeling the emotions of music and listening to music. This is why many people prefer to go to concert, since concert music is very different from records and recorded music. Audiences can feel the atmosphere of music on the spot, watch performers' body language and facial expression and feel the temperament of the stage, etc. These are all ways to enrich music; meanwhile, they are important constituents of music, and audiences can absorb more music nutrition from it.

Cello players' body language can not only present unique music language, but also can present players' inner world. Body language is an approach for cello players to express their feelings, just like writers use words to express their emotions and present their inner world. When people are reading literature, they can walk into writers' inner world by beautiful words and feel writers' inner feelings. Likewise, when people are appreciating music, they can also feel players' inner emotions 
by beautiful rhythm, their movements and facial expressions, walk into players' inner world and touch players' artistic soul. Based on the important functions of body language, cello players can help audiences appreciate music better by suitable and beautiful body language and facial expression, and it would be easer for them to have resonance with audiences.

If cello players want to attract audiences more, they can first catch audiences' attention by body language, and then use music so that music can not only stay on auditory layer, but also strengthen visual feelings. Therefore, some accurate and necessary body language can not only add brilliance to cello players' splendor, but also can help audiences understand music better. Conductors conduct music performance by body language. Maybe audiences do not understand conductors' movements much, but they can also feel changes of music by conductors' gesture and movements and recognize the strength and ups and downs of music, thereby understanding music better. Good body language is an effective way for cello players to lead audiences into the artistic conception of cello; meanwhile, body language is a resonance of natural state for cello players and music.

\section{Characteristics of body language in cello performance}

Body language in cello performance relies on players themselves more, and it is not as abundant as the body language of dancers; rather, it is restricted much. On the one hand, cello players are restricted by cellos; on the other hand, they are restricted by music. The body language of cello players must be based on completing the performance and guaranteeing the quality of performance, and body language can only work as the assistance; it cannot steal the show or be too exaggerated. If cello players pursue the presentation of body language too much, the tone, intonations and so on would be influenced. Besides this, because of the characteristics and style of cello, exaggerated body language might not be applicable in cello performance. Players' body language must suit the style of the music and conform to the emotional intention of music. Cello players should grasp these restrictions. They cannot be too exaggerated or conservative. Only in this way can audiences' resonance be stimulated and their antipathy be avoided.

The size of cello is huge, so players would be under many restrictions. They cannot have wide movements like players of small-sized instruments. Cello players must rely on the cello foot for support and immobilization, and they must sit up straight for better performance. Therefore, cello players' bodies are restricted much during performance; their movement range must be small, and most of their main movements are on the head, face and neck. Though cello players' body language is restricted much, small range of body language is sufficient to express emotions. Therefore, many excellent cello players have small range of body language, but their profound understanding of music still touch audiences deeply.

Body language of cello players also has distinct individual differences. Because of different height, weight and gender, cello players might also be restricted in their body language. No matter what individual differences cello players have, their premise for performance is to guarantee the quality of music. Therefore, they must concentrate their power on their back and observe basic movements of cello performance. Excellent cello players can complete the performance of music under these restrictions and present unique body language style. The victory movement of cello players is very important; it is the presentation of the consistency of muscles and it can relax the muscles. If cello players want to present natural body movements, they need to learn how to send power from the back to arms, hands and fingers; meanwhile, they can give full play to it according to specific situations. In this way, they can make body language and the music consistent and coherent, thereby forming their unique performance style. 


\section{Methods for cello players to apply body language appropriately}

If cello players want to apply body language appropriately, they must have sufficient understanding of the music work and understand the music style, and they must have profound research on the subjects and creation background of the work as well as composers' creation motivation. For example, when performing Bach's Cello Suites, performers must know that this is a piece of music with religious characteristics. Besides presenting the composer's extraordinary talents, the music should also show the composers' belief. Therefore, when playing this piece of music, the players' body language should reveal solemn feelings; their facial expressions should not be frivolous; rather, they should be dignified and devout to show their respect to the composers' belief. Many cello beginners' understanding of the work is too superficial, so it is easy for them to make inappropriate body language. Therefore, if players want to present body language better, they must have profound understanding of the music work. In this way, they can have resonance with music in the performance process, and the facial expressions they reveal as well as the body language they show can fit with the work more.

Cello players' understanding of music is also progressive. Only by studying continuously can they can have better understanding of music works from different times and make no mistakes in the performance process, which would lead to wrong performance ways and inappropriate body language and facial expressions. Many senior artists still have strict demands on themselves; they believe that their understanding of music is still not accurate enough and they still need a long time for comprehension and disintegration to fully figure out problems in performance; only at this time can they have deeper understanding of the music. This process of progressive understanding is very precious and happy. Therefore, cello players cannot be satisfied with current understanding; rather, they should pursue continuously for more perfect agreement with music.

The presentation of cello players' body language is also closely related to their own temperament. Therefore, cello players must concentrate on the improvement of their own inner culture to present more perfect integrate image. Players with more profound inner culture usually present more natural and coherent body language in performance and give people enjoyment of beauty. It is just like writing. If a person wants to improve writing skills, they not only have to dig into rhetoric and literary quotation; rather, they should also be equipped with profound knowledge. It is the same with cello performance. Players need to absorb more knowledge besides performance, absorb nutrition from other art and enrich their inner heart. When it is accumulated to a certain degree, a qualitative leap would happen; in the meantime, they can digest various artistic forms and be more calm and generous in the performance process. Also, they would have deeper understanding of music and their expression of music would be more accurate and vivid.

For the same piece of music, different performers would present different styles and temperament. Senior players would be more serious, stable and solemn, while younger players would be more smooth and relaxed. Though their styles and body languages are different, they are all based on their own understanding of the work. Cello players can choose their own preference and style according to their own understanding. However, all excellent cello players deepen their own understanding by improving their inner culture. In this way, when they are equipped with performance techniques, they can also integrate with the music and reach more perfect realm. Just because of their strict demands on themselves and continuous pursuit for art, cello players can make their performance effects better and explain the works more perfect; in the meantime, by continuous improvement of their own artistic culture, their unique body language and performance styles are formed, adding splendor to cello works. Cello players' culture is critical for the application of body language. Therefore, cello players need to be strict with themselves at ordinary times, study and 
improve themselves continuously and enrich their own heart and feelings. Only in this way can they have deeper understanding of the work and present body language with their unique styles at the same time.

\section{Conclusion}

In conclusion, body language with beautiful posture, easy grace and combination of movement and inertia is a very important part in the performance of cello. By players' body language, not only players' styles can be presented, but also their inner culture can be better shown. In the meantime, it can help audiences get into the musical realm better, so it is a communication bridge between audiences and players. If cello players want to present body language better, they must apply it appropriately and reasonably to reach perfect unity between music, movement and facial expressions. To reach this goal, cello players need to have sufficient understanding of the work and pay attention to the improvement of their own inner culture. In this way, they can bring aesthetic enjoyment to audiences and cello performance can bet more vivid and perfect.

\section{Bibliography:}

[1]Chen Meng. On Body Language During Cello Performance [J]. Art Education,2016,(08):118-119.

[2] Mai Chenduo. On Tension and Relax of Left and Right Hands During Cello Performance [J]. Inner Mongolia Art,2016,(01):18-19.

[3] Zhao Na. On Art Expressive Force During Cello Performance [J]. Home of Theatre(First Half),2012,(06):31.

[4] Yang Xuexin. On Problems During Cello Performance [J]. Journal of Kiamusze Educational College,2010,(03):163+195.

[5] Zhang Jiaohua \& Yu Yaochun.On the Cultivation of Expressive Force During Cello Performance [J]. Music World,2005,(09):49-50. 